\title{
Multi-axis dynamic displacement measurement based on a strain shunt structure
}

\author{
Qingfeng $\mathrm{Xia}^{1}$, Haihu $\mathrm{Liu}^{2 *}$ \\ ${ }^{1}$ Department of Engineering Science, University of Oxford, Parks Road, Oxford, OX1 \\ 3PL, United Kingdom \\ ${ }^{2}$ School of Energy and Power Engineering, Xi' an Jiaotong University, 28 West \\ Xianning Road, Xi'an 710049, China \\ *E-mail: haihu.liu@xjtu.edu.cn
}

\begin{abstract}
:
Transient gap or crack width monitoring is essential for structural health monitoring and failure analysis of large civil structures. In this paper, an innovative multi-axis displacement sensor, which utilises metal foil strain gauges on a strain shunt structure, has been proposed. This displacement sensor has the advantages inherited from metal foil strain sensing, such as low cost, high precision, fast dynamic response and low power consumption, and can also measure displacement in two axes independently. The working principles and sensitivity are derived theoretically from key geometrical parameters of the shunt structure, and the linear response of strain values to the given displacement of two translational axes has been demonstrated experimentally. Furthermore, modal response, stress concentration, optimal gauges installation positions and bending deformation due to moment of the third axis are studied numerically.
\end{abstract}

Keyword: Displacement sensor; crack monitoring; strain gauge; SHM; wireless sensor network

\section{Introduction}

The trend of Internet of Things (IoT) has a profound impact on asset management, because the deployment of interconnected sensors has the potential to optimise the resource usage and thus maximise the economic performance. For example, structural health monitoring (SHM) of a wind turbine foundation is critical to health assessment [1], which can not only minimise downtime but also reduce huge 
maintenance and logistics impact [2, 3]. To monitor these less accessible off-shore platforms, sensors must be rugged, robust, light-weighted, and less energy-thirsty for wired and wireless sensor networking operating in harsh environments. This paper highlights the necessity of low-cost multi-axis displacement sensor that could perform precise dynamic measurement of surface crack variation and gap change.

A variety of contact and non-contact sensors can measure displacement and position, including those based on electromagnetic variation, mechanical deformation, and optical/visual/acoustic solutions, and so on. The first category relies on the linear variation of electromagnetic quantity with displacement, and the electromagnetic quantities generally include resistance and capacitance, eddy current, and magnetic induction. Potentiometer with a sliding contact on an adjustable resistor provides a linear relationship between distance and resistance, but its application is limited by wear, low precision and slow dynamic response. Highly precise capacitance displacement sensor, utilising a charge amplifier and alternative current circuit to measure the capacitance change, is vulnerable to contamination on the target surface. Magnetic induction based solutions, such as linear voltage displacement transducer (LVDT) and eddy current sensor, need cores to generate magnetic field. Frictionless LVDT is claimed to have a good dynamic response, and limited only by the inertial effect of the probe. However, frictionless LVDT is risked by wear due to the displacement in off-design direction. Eddy current sensor has certain tolerance of nonconductive contamination, but its accuracy is still affected by the conductive property of target surfaces. In addition, these sensors are all sensitive to external electromagnetic interference, and thus they cannot be used near motors, generators, and permanent magnets and other places with high electro-magnetic interference.

Optical, acoustic and visual solutions involve wave sources or digital devices for the displacement measurement, such as laser or ultrasound range finder, optical fibre, laser confocal, image correlation, and digital calliper. Optical and visual solutions are ideal for a large displacement measurement, while time-of-flight based acoustic solution can provide precise measurement of displacement or thickness for a low wave speed. Time-of-flight based optical displacement sensor is less precise for the small measurement range, due to the challenge in measuring small temporal difference. On the other hand, a vision-based displacement sensor, which relies on 
image capture equipment and image processing algorithm, can measure dynamic response up to ten $\mathrm{Hz}$ [4] at the cost of a fast vision capture equipment and a high performance computing unit. In addition, a cheaper solution based on optical mouse is capable of measuring two-dimensional displacement [5], but the cost and power consumption are admittedly discouraging for wireless sensor networking.

Displacement/gap sensor based on strain gauge, which takes advantage of the mature metal foil strain gauge technology, has many advantages including high precision and power efficiency [6] and the ease of data acquisition [7]. Most of strain gauge based solutions utilise single cantilever beam, converting the deflection deformation on the free end into strain measurement on the fixed end. A typical Vishay single axis displacement sensor using strain gauges has a dynamic response of $100 \mathrm{~Hz}$ for the 5 $\mathrm{mm}$ displacement range[8]. Furthermore, the energy-efficient strain gauge excitation circuit is crucial for battery-based wireless sensor networks. Compared with the sensors using electro-magnetic field excitation circuit, the power consumption of a strain gauge based sensor is significantly reduced.

For the specific application of measuring relative movement between components or surface crack monitoring for structures under dynamic and multi-axis load, a displacement sensor with high precision, high dynamic response but low cost is highly desired. For example, the cracked parts of a concrete foundation may undergo three-dimensional relative displacement. Non-destructive detection techniques, such as ultrasound-based instrument, have the potential to evaluate internal cracks, but the quantification of crack information and failure analysis is very challenging without insitu dynamic measurement. Mohammad and Huang [9] highlighted the necessity of direction information during crack monitoring, in order to analyse the magnitude and direction of the underlying actuating forces.

Surface-mounted multi-axis displacement sensors are useful to evaluate the severity and cause of major cracks on infrastructure, when embedded sensors like fibre optic strain gauges [10] are not available. In the scenario of long-term structure health monitoring, the electromagnetic excited displacement sensors are discouraging due to high power consumption in wireless sensor networking and high cost for multiple axes displacement measurement. Photography and image processing have been 
widely utilised for the long-term crack evolution study [11], in which the twodimension (2D) displacement is measured through a single camera setup. However, the frequency response is limited by the camera frame-rate and computational resource of embedded systems for image processing, and meanwhile the precision is constrained by the pixel resolution. Nevertheless, the crack separation in the surface normal direction, which is essential to detect the shearing force, cannot be observed by the single camera setup.

The recently-developed strain shunt method for the large dynamic strain measurement can be adapted to measure dynamic displacement precisely $[12,13]$. A strain shunt structure consists of a straight sheet and at least two strain relieving units, e.g. cantilevers. It can be fabricated in a metal sheeting process using a single piece of metal sheet, which enhances the dynamic response of the sensor. Unlike a clip gauge structure, the distinct responses to stretching and bending deformation of the attached base plane have been identified [12], and a redesigned strain relieving unit and a shunt sheet as close as possible to the target surface can reduce the difference in responses. A displacement sensor based on the strain shunt structure is superior to the single cantilever configuration for a small footprint, compared with the setup of cantilever in micro-scale sensing [14]. With the mature technique of metallic film strain gauge, the displacement sensors based on strain measurement have the strengths of high precision, low cost, low power consumption and sufficient dynamic response for wireless sensor networking. Furthermore, it is possible to measure multi-axis displacement simultaneously via a combination of several strain sensing units on different strain shunt positions. The reduced footprint and simplified installation are preferred for the applications in condition monitoring and SHM.

In this paper, the working principle of a displacement sensor based on strain shunt structure with $\cap$-shaped strain relieving units is demonstrated theoretically and experimentally. The sensitivity between the strain measurement and displacement is predicted via a theoretical analysis, and a prototype of this multi-axis displacement sensor is experimentally validated against a digital dial gauge. Furthermore, the sensitivity to multi-axis displacement, stress concentration and optimal strain gauge installation position are investigated by the finite element analysis, targeting on a higher technology readiness level. 


\section{Principles of strain-based displacement sensor}

\subsection{Existent design of strain based displacement}

A cantilever beam can convert deflection displacement into measurable strain on surface. In contrast to compressive or tensile force acting on a cantilever beam, the bending force can generate considerable flexural deformation on the free end. For example, a moving displacement sensor probe connecting to a cantilever beam can cause strain variation on the beam surface, as shown in Fig 1 (a). Strain gauge is installed on the surface near the fixed end where the surface strain is higher, in order to measure the applied displacement.

The deflection of a simple cantilever under bending force, is expressed by Euler beam theory for a slender beam [15],

$$
\delta_{b}^{E u l a r}=\frac{F H^{3}}{3 E I}
$$

where $E$ is the elastic modulus; $F$ is the magnitude of bending force acting at the free end; $H$ is the height; $I$ is the second moment inertia for the cross-section of the cantilever beam. It should be noted that the bending displacement predicted from Euler beam theory is applicable only to slender beams, where the thickness to height ratio is generally small.

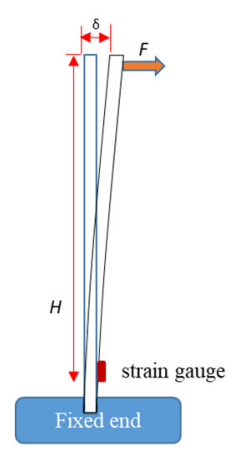

(a)

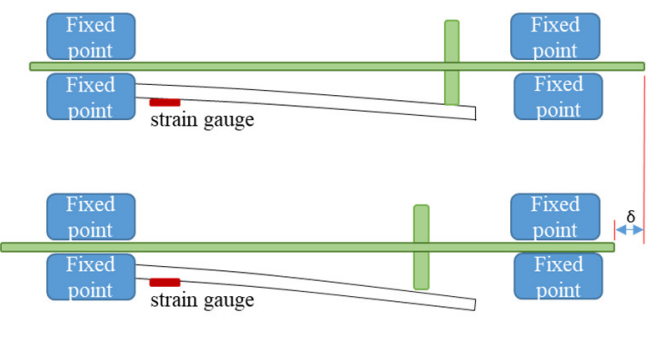

(b)

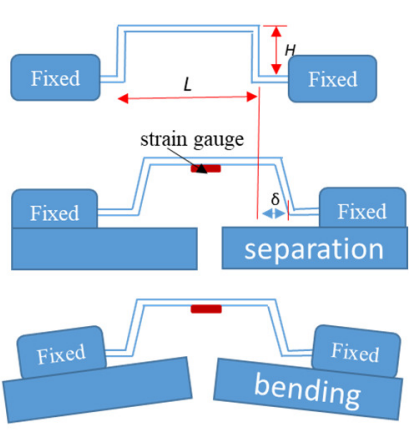

(c)

Fig. 1 Schematic of strain gauge based displacement measurement: (a) cantilever, (b) spring probe, and (c) clip-gauge.

The second design of displacement sensor shown in Fig. 1 (b) has a reduced footprint, but the performance of dynamic response may be undermined. This kind of 
displacement sensor has a similar probe design as LVDT sensors; the sensor probe is preloaded by a spring to follow the target surface. Strain gauges are installed on the cantilever beam nearly parallel to the displacement sensor probe, and the sensor height is significantly reduced in contrast to the simple design in Fig. 1 (a). However, the sliding contact between the sensor probe and cantilever limits its application to low speed scenario.

The third design shown in Fig. 1 (c) is based on a clip gauge structure; the deformation on the target surface leads to strain variation on the structure captured by strain gauges. A clip gauge is proposed to measure large dynamic strain on the target surface via scaling down the strain value to a level manageable by a metallic foil strain gauge [12]. Given a sufficiently small scaling ratio, the clip gauge can measure the gap variation between fixing legs. However, it is found that the stretching and bending deformations on the target surface lead to distinct scaling ratios, and thus distinct displacement derivations from the strain measurement [12]. Therefore, the demonstrated clip gauge design with only one strain sensing unit in Fig. 1 (c) cannot measure the displacement between two connection points, if both bending and stretching deformations exist. Therefore, an innovative structure known as strain shunt is proposed to measure multi-axis displacement and bending moment between two joints simultaneously.

\subsection{Displacement sensor based on strain shunt}

Strain shunt structure, originally designed to measure large dynamic strain via proportional scaling-down [13], can be adapted to measure small but highly dynamic displacement. One possible design of strain shunt structure, consisting of a straight sheet and two $\cap$-shaped units, is illustrated in Fig. 2 (a). Compared with the existing designs in Fig.1, this new displacement sensor based on strain sensing can measure the displacement in more than one direction and avoid the sliding contact between probe and cantilever. Non-sliding contact guarantees the dynamic performance and precision.

The straight and flat sheet parallel to the surface underneath is denoted as shunt sheet, and it is close to the target surface as much as possible in order to minimise the 
scaling ratio difference in response to stretching or bending deformations on the target surface. Strain gauges are installed on the shunt sheet to measure the $\mathrm{x}$-axis displacement between two binding feet, i.e. along the shunt sheet direction. Given an $\mathrm{x}$-axis separation between two binding feet, most of the deformation occurs on the strain relieving units, i.e., the $\cap$-shaped cantilevers in Fig. 2. Multiple strain relieving units in series can provide large displacement measurement range, but the performance of dynamic response is undermined. Furthermore, extra strain gauges can be installed on the $\cap$-shaped cantilevers to sense the $y$-axis displacement, as shown in Fig. 2(c). This strain shunt structure is fabricated from one piece of steel sheet via the metal sheeting process, in order to achieve faster dynamic response. The shunt structure can be easily bonded to the target surface by either fastening or adhesion.
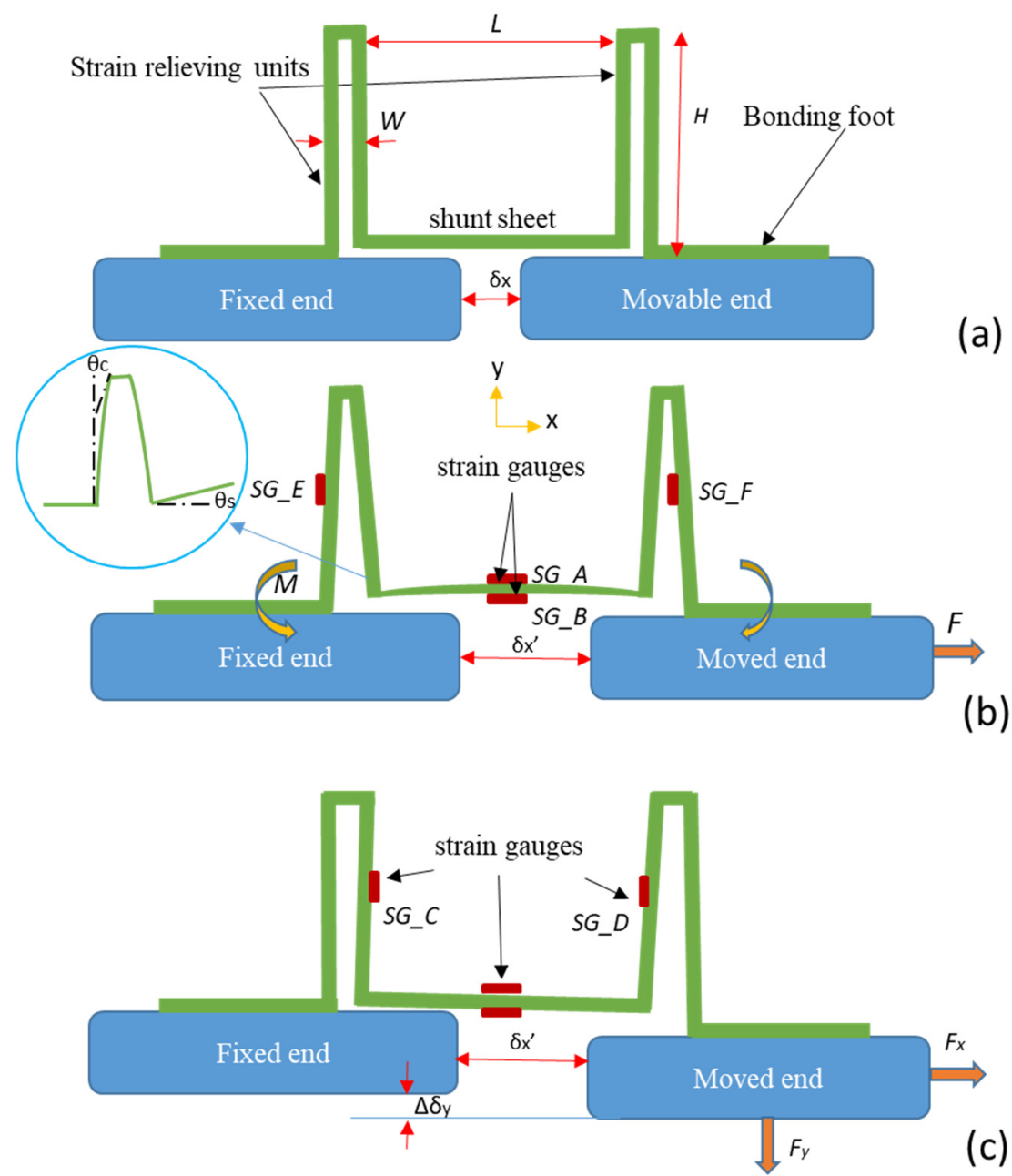

Fig. 2 Schematic of the displacement measurement by strain shunt structure (a) without deformation; (b) with an $x$-axis displacement only; (c) with a combination of $x$-axis and $y$-axis displacements. 
As illustrated in Fig. 2 (b), the applied displacement is distributed to $\cap$-shaped units with a height $H$ and the shunt sheet with a length $L$. One $\cap$-shaped strain relieving unit can be simplified into 2 cantilevers under bending load. The deflection angle of single cantilever is denoted as $\theta_{c}$, and the deflection angle of the shunt sheet is $\theta_{s}$ is illustrated in Fig. 2 (b). Due to the elastic deformation of cantilever under load, the deflection of $\cap$-shaped beams relieves the displacement (strain) on the shunt sheet. For the one-dimensional separation $\Delta \delta_{x}$, the deformation is distributed as

$$
\Delta \delta_{x}=4 N \delta_{c}+\delta_{s}
$$

where $N$ is the number of $\cap$-shaped strain relieving units on each side of shunt structure and $N=1$ is studied for the convenience of experimental verification; $\Delta \delta_{\mathrm{x}}$ is the $\mathrm{x}$-axis deformation measured between the binding feet; $\delta_{c}$ is the $\mathrm{x}$-axis deformation of each supporting cantilever; $\delta_{s}$ is the $\mathrm{x}$-axis deformation of the shunt sheet. Notably, the fillet radius and gap between bottom surface and shunt sheet are neglected in this one-dimensional analytical model, and the Euler beam theory is used for the slim beam in this strain shunt design.

In order to measure small dynamic displacement by a strain shunt structure, a hypothetical strain $\varepsilon_{i}$ is defined as the ratio of length variation to span between the binding feet, i.e. $\Delta \delta_{x} /(L+2 W)$. Since multiple supporting cantilevers are subject to deformation, the strain on the shunt sheet should be less than the hypothetical strain. The ratio of the averaged x-axis strain on the shunt sheet $\varepsilon_{s}$ to the hypothetical strain $\varepsilon_{i}$ is denoted as shunt ratio $r_{s}$, i.e.

$$
r_{s}=\frac{\varepsilon_{s}}{\frac{\Delta \delta_{x}}{L+2 W}}=\frac{(L+2 W) \varepsilon_{s}}{\delta_{x}^{\prime}-\delta_{x}}
$$

where $\delta_{x}$ is the initial gap between separate components, and $\delta_{x}^{\prime}$ is the gap after deformation. As a result of the bending moment $M$ introduced at the joint of the shunt sheet and fixture surface, the shunt sheet warps correspondingly. In particular, the strain values on the upper and the lower surfaces of the shunt sheet are significantly different due to the warping deformation of the shunt sheet. Therefore, $\varepsilon_{s}$ is calculated from the average strain on the upper and lower surfaces of the shunt sheet. 
Once the scaling ratio $r_{s}$ is analytically or experimentally derived and the average strain on the shunt sheet is measured, the variation of the shunt structure span for single strain relieving unit on each side, i.e. the displacement change $\Delta \delta_{x}$, can be obtained as

$$
\Delta \delta_{x}=(L+2 W) \varepsilon_{i}=(L+2 W) \varepsilon_{s} / r_{s}
$$

\subsection{Derivation of displacement sensitivity}

In order to predict the displacement from strain gauge measurement on the shunt structure, the shunt ratio needs to be derived analytically according to Eq. (2). In addition to the warping effect, the deformation of the shunt sheet is caused by the tensile or compressive force $F$ as a result of the x-axis displacement, i.e.

$$
\varepsilon_{s}=\frac{F}{A_{s} E}
$$

where $A_{s}$ is the cross-section area of the metal sheet folded into a shunt structure.

Meanwhile, the warping shunt sheet has a significant impact on the cantilever deflection, if the height of cantilevers $H$ is of the same order of magnitude as the shunt sheet length $L$. The bending moment $M$ at the joint of cantilever and shunt sheet, induced by the $\mathrm{x}$-axis displacement, decreases the deflection of the supporting cantilever.

$$
\delta_{c}=\frac{F H^{3}}{3 E I}-\frac{M H^{2}}{2 E I}
$$

where $I=b^{3} w / 12$ is the second moment of inertia for a metal sheet cross-section with thickness $b$ and width $w$.

Although the moment $M$ acting on the shunt sheet joint is unknown, it could be derived from the assumption that the joint angle between the vertical surface of the cantilever and the shunt sheet remains constant during deformation. With such an assumption, the warping angle of the shunt sheet $\theta_{s}$ is approximated as the sum of 
deflection angles of the $\cap$-shaped strain relieving units at the joint (see Fig 2(b)). For $N=1$, it is twice the deflection angle of single straight supporting cantilever $\theta_{c}$.

$$
\theta_{s} \approx 2 \theta_{c}
$$

Note that Eq. (7) cannot be generalised to describe multiple strain relieving units, since the assumption of a constant angle at the joint may lead to substantial errors for $N>1$. This assumption will be justified in numerical prediction of shunt ratio. In Eq. (7), the deflection angle $\theta_{c}$ is obtained by subtracting the deflection angle induced by the bending moment $M$ from the angle derived from Euler beam theory for a cantilever with one end fixed and the other end under bending force $F$ :

$$
\theta_{c}=\frac{F H^{2}}{2 E I}-\frac{H M}{E I}
$$

On the other hand, the shunt sheet is modelled as a beam fixed on both sides, and the bending moment $M$ acting on both ends of the shunt sheet results in the deflection angle $\theta_{s}$. Given the large ratio of length to thickness for the shunt sheet, the classic Euler beam theory can be used to calculate the warping angle $\theta_{s}$ for the shunt sheet with $M$ on both ends, i.e.,

$$
\theta_{S}=\frac{M \frac{L}{2}}{E I}
$$

where the beam length is the half length of the shunt sheet span [16] since the centre of the shunt sheet is horizontally-symmetric without deflection angle.

Substitution of Eqs. (8) and (9) into Eq. (7) leads to,

$$
2 \theta_{C}=2\left(\frac{F H^{2}}{2 E I}-\frac{H M}{E I}\right) \approx \frac{M \frac{L}{2}}{E I}
$$

which further gives an estimate of the bending moment as $M=2 \mathrm{FH}^{2} /(4 H+L)$. Evidently, this bending moment can cause considerable warping deformation and an extra strain on the thin shunt sheet surfaces, which is defined by 


$$
\varepsilon_{w}=\frac{\left(\frac{b}{2}\right) M}{E I}=\frac{b F H^{2}}{(L+4 H) E I}
$$

Once the warping angle is calculated, the x-axis displacement of the shunt sheet incurred by warping can be derived. The shunt sheet is deformed into an arc shape with the deflection angle $\theta_{s}$ on each end, and the reduction in $\mathrm{x}$-axis length of the shunt sheet induced by warping is

$$
\delta_{x_{-} w}=\left(\frac{\theta_{s}-\sin \theta_{s}}{\theta_{s}}\right) L \approx \frac{\theta_{s}^{2}}{6} L
$$

which is one order smaller than $\delta_{c}$. Therefore, its impact on the averaged shunt ratio can be neglected.

Eventually, Eq. (2) can be rewritten as

$$
4\left(\frac{F H^{3}}{3 E I}-\frac{M H^{2}}{2 E I}\right)+\frac{L F}{A_{S} E}=\frac{1}{r_{S}} \frac{L F}{A_{S} E}
$$

in the case of $N=1$. Since the shunt structure made of a single piece of metal sheet, the elastic modulus, thickness and width of the metal sheet are all constants, the shunt ratio can be derived from Eq. (13):

$$
r_{S}=\frac{\frac{L}{A_{S} E}}{4\left(\frac{H^{3}}{3 E I}-\frac{H^{2}}{(4 H+L) E I}\right)+\frac{L}{A_{S} E}}=\frac{L b^{2}}{16\left(1-\frac{3 H}{(4 H+L)}\right) H^{3}+L b^{2}}
$$

The above analytical formula shows that the shunt ratio is independent of material properties and the direction of tensile/compressive forces acting on the shunt sheet, when assuming rigid connections between the bonding feet and the target surface. In practice, the thermal expansion coefficient of the shunt structure is similar to that of the target surface, and thus the impact of thermal expansion coefficient can be minimised. The warping deformation of the shunt sheet leads to different strain values at the centres of both upper and lower surfaces, where strain gauges are installed. The shunt ratios based on the strain at the centres of the upper or lower surface are given by 


$$
\begin{aligned}
& r_{S_{-} \text {upper }}=r_{S}\left(1+\frac{\varepsilon_{w}}{\varepsilon_{S}}\right)=r_{S}\left(1+\frac{12 H^{2}}{(L+4 H) b}\right), \\
& r_{s_{-} \text {lower }}=r_{S}\left(1-\frac{\varepsilon_{w}}{\varepsilon_{S}}\right)=r_{S}\left(1-\frac{12 H^{2}}{(L+4 H) b}\right)
\end{aligned}
$$

Since the metal sheet thickness $b$ is substantially smaller than the height of the shunt structure $H, r_{s_{-} \text {upper }}$ may have an opposite sign to $r_{s_{-} l o w e r}$.

In the application of displacement measurement, the difference of strain values at the lower and upper centres is measured by a half-bridge gauge configuration which can effectively cancel thermal strain due to temperature rise. Because the shunt ratio is small, typically less than 0.001 , for displacement sensing, the displacement sensitivity along $\mathrm{X}$-axis can be defined by the strain difference as

$$
\frac{\varepsilon_{A}-\varepsilon_{B}}{\Delta \delta_{x}}=\frac{\varepsilon_{i}\left(r_{s_{-} \text {upper }}-r_{S_{\text {lower }}}\right)}{\Delta \delta_{x}}=r_{S} \frac{24 H^{2}}{L(L+4 H) b}
$$

where $\frac{24 H^{2}}{L(L+4 H) b}$ is denoted as warping ratio in this study. According to Eqs. (13) and (16), the sensitivity can be controlled by key geometrical parameters, while the displacement range is limited by fatigue limit of the shunt sheet material.

The displacement sensor based on strain shunt design can measure two components of 3D translational displacement. As shown above, the strain variation induced by the $\mathrm{x}$ axis displacement can be analytically obtained. We will describe in Section 4 how the $\mathrm{y}$-axis displacement is numerically evaluated. Sensing units should be insensitive to the displacement in the third direction, i.e. z-axis, if all the strain gauges are perfectly installed at the centre of the steel sheet. To note, it is possible to measure 3-axis displacement using 2 such strain shunts based sensors. Two displacement sensors with the angles of 45 degrees and - 45 degrees to the crack line can be installed, similar to shearing strain measurement using 2 strain gauges with an angle of 90 degrees.

Concerns should be taken for the strain-based displacement sensor, such as creep and fatigue. Regular calibration or zero-offsetting is necessary for long-term monitoring, which is a common practice for any sensor using metal foil strain gauges. Calibration 
is unnecessary for transient displacement measurement, because the sensitivity coefficient only depends on key geometrical parameters and it is less affected by fatigue. Fatigue failure of shunt structure can be avoided by carefully selecting geometrical parameters according to the desired displacement range. A further reduction of stress concentration can mitigate the risk by limiting the maximum stress under the material fatigue limit. Besides, the shunt structure has an intrusive impact on the target system for the bonding installation, while the force introduced by the metal sheet is negligible for large components typically found in structural heath monitoring.

\subsection{Dynamic response}

For the displacement sensor under dynamic load, the first harmonic frequency of the shunt structure must be significantly higher than the variation frequency of the displacement to be measured. For the strain shunt structure in Fig. 2 (b) under x-axis displacement, the resonance frequency $f$ can be predicted theoretically by

$$
f=\frac{1}{2} \sqrt{\frac{k}{m}}
$$

when the single spring system has no mass at the end. Herein, $k$ and $m$ are the stiffness and mass of the spring, respectively. For the current shunt structure design, the stiffness can be expressed as

$$
k=\frac{F}{\Delta \delta_{x}}=\frac{E A_{s}}{L} r_{s}
$$

Since the stiffness is determined by key geometrical parameters, the desired dynamic response can be taken into account during the design stage. In addition, due to the small thickness to length ratio of the shunt sheet, the warping or deflection actuation leads to a much smaller resonance frequency, which will be discussed in the subsequent numerical study.

\section{Experimental validation}

\section{1 Experimental setup}


The schematic diagram of experimental setup for the proposed displacement sensor and the close-up of the displacement structure with strain gauges are shown in Fig. 3. One foot of the shunt structure moves with the 3D traverse, relative to the fixed foot on the other end. The displacement can be measured precisely by a Mitutoyo digital dial gauge, with a precision of $0.001 \mathrm{~mm}$. The relationship between the strain value and $3 \mathrm{D}$ displacement is to be investigated experimentally.

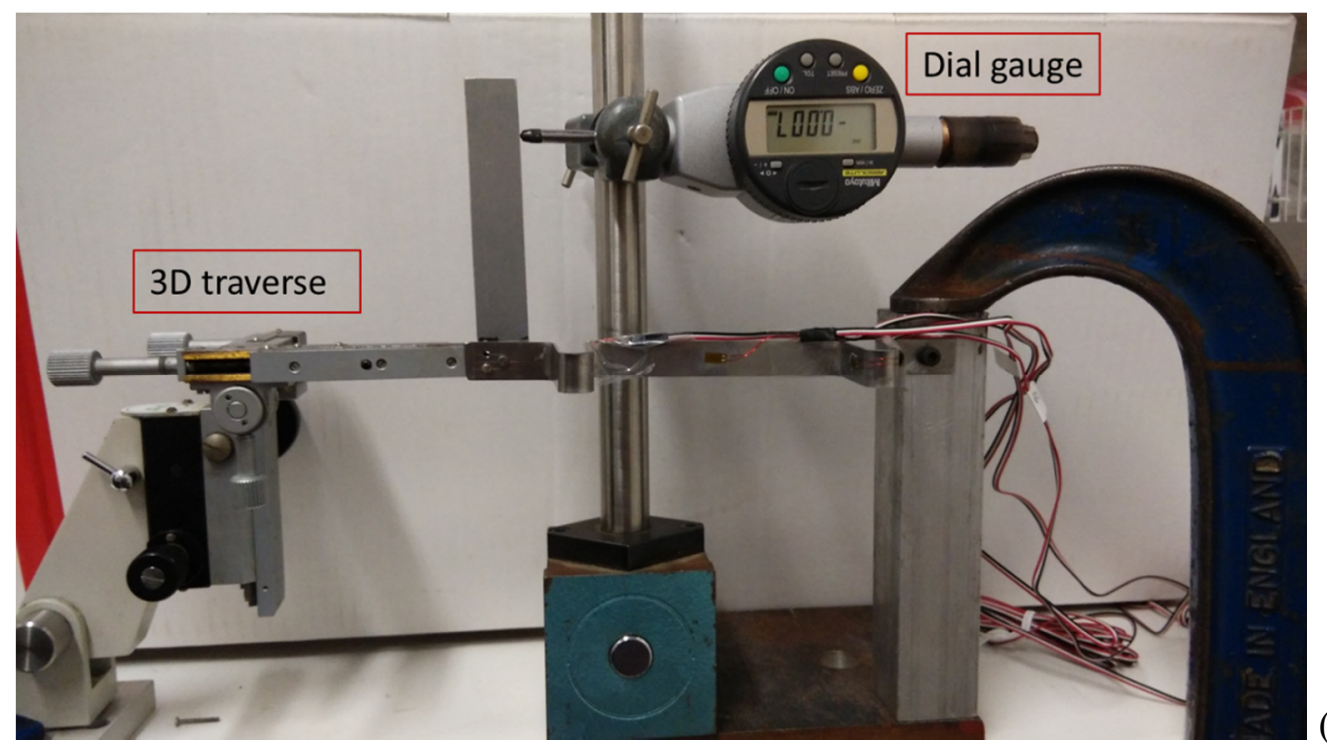

(a)

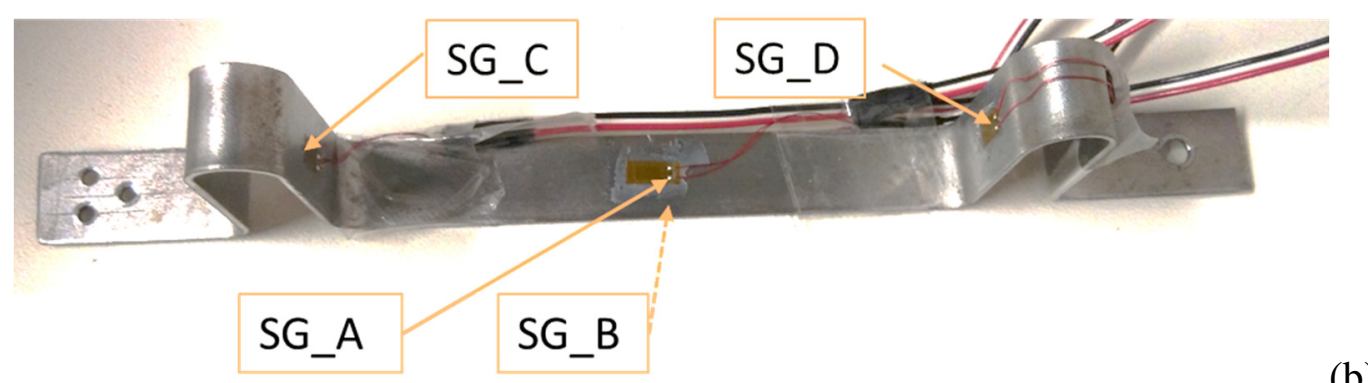

(b)

Fig. 3 (a) Experimental setup for static displacement measurement; (b) strain gauge positions.

Medium carbon steel is used to fabricate the strain shunt structure because of its high yielding strength. The shunt structure has a thickness of $1.06 \mathrm{~mm}$ and a width of 13 $\mathrm{mm}$. The average height of the shunt sheet $H$ is about $34.5 \mathrm{~mm}$, which is measured from the sheet centre line; the span of the shunt sheet is $L=91 \mathrm{~mm}$, and the width of $\cap$-shaped turns is $W=11 \mathrm{~mm}$. This strain shunt structure is made by the metal sheeting process, which is convenient to manufacture such a structure in large volume. The inner fillet radius is controlled as $5 \mathrm{~mm}$ for the joint of cantilever at the $\cap$-shaped turn 
and $2.5 \mathrm{~mm}$ at the binding feet. The large fillet radius can reduce the stress concentration near the turns, as identified in the previous strain shunt design [6].

Two metal foil strain gauges of type Vishay C2A-06-125LW are installed at the centres of the lower and upper surfaces of the shunt sheet, which are shown as SG_A and SG_B respectively in Fig. 3 (b). To obtain the $\mathrm{x}$-axis displacement and validate the analytical model, it is necessary to measure the strains on SG_A and SG_B in the middle of the straight shunt sheet. Extra strain gauges are applied onto the strain relieving units (SG_C and SG_D in Fig. 3 (b)), in order to detect the displacement in the $y$-axis direction. Dimension of the effective gauge grid is $1 \times 2 \mathrm{~mm}$ for this type of strain gauge, while the overall dimension is $2 \times 4 \mathrm{~mm}$ with an extra area for epoxy binding. Quarterly-bridge is precise in the constant temperature laboratory environment, and it is confirmed by matching the difference in the measurement of two gauges with the output of a half-bridge configuration for these gauges. In practice, the half-bridge configuration of SG_A and SG_B should be used to cancel the temperature induced variation, and to eliminate the effect of displacement in another direction. The voltage signal from the strain gauges is amplified and digitalised by a wireless sensor network strain gauge module NI-WSN 3214 supplied by National Instrument, which has a high analogue to digital conversion resolution (ADC) of 20 bits. Finally, the digitalised voltage signal is gathered by an Ethernet gateway NIWSN 9791 for further data processing. At least 200 samples are recorded for each displacement position, at a sampling rate of 10 samples per second. The temporal standard variation of strain measurement is found to be $1 \mu \varepsilon$.

\subsection{Experimental validation}

The linear relationship between the strain values on the present shunt structure and the $\mathrm{X}$-axis displacement is experimentally validated in Fig. 4 (a). $\varepsilon_{A}$ and $\varepsilon_{B}$ are the measured $\mathrm{x}$-axis strain values for $\mathrm{SG} \_\mathrm{A}$ and $\mathrm{SG} \_\mathrm{B} ; \varepsilon_{C}$ and $\varepsilon_{D}$ are the measured $\mathrm{y}$-axis strain values for SG_C and SG_D. The sensitivity coefficient in the X-axis direction, defined as the slope of the fitting line in a plot of strain difference $\varepsilon_{A}-\varepsilon_{B}$ versus X-axis displacement, is $305 \mu \varepsilon / \mathrm{mm}$. Results of both positive and negative $\mathrm{x}$-axis displacements are presented, and an insignificant hysteresis is observed in Fig. 4 (a). The hysteresis error is attributed to the hysteresis of the 3D traverse system, and the 
measurement uncertainty of the dial gauge and strain gauges. On the other hand, $\varepsilon_{C}-\varepsilon_{D}$ is supposed to be insensitive to the $\mathrm{x}$-axis displacement, but the positional error of strain gauge installation leads to a linear response of $\varepsilon_{C}-\varepsilon_{D}$ to the $\mathrm{x}$-axis displacement. The sensing coefficient is $12.2 \mu \varepsilon / \mathrm{mm}$ for the current experimental setup, which should be minimised by the reduced gauge positioning error via an improved gauge installation method. Gauge pattern pre-printed on the metal sheet might be a choice.

Fig. 4 (b) confirms that the strain difference $\varepsilon_{C}-\varepsilon_{D}$ linearly increases with the y-axis displacement, while $\varepsilon_{A}-\varepsilon_{B}$ is not sensitive to the y-axis displacement. The sensitivity for the $\mathrm{y}$-axis displacement, obtained by the linear fitting of $\varepsilon_{C}-\varepsilon_{D}$ against $\mathrm{y}$-axis displacement, is $86.6 \mu \varepsilon / \mathrm{mm}$. As shown in Fig. 4 (a) and (b), the hysteresis error of $\varepsilon_{C}-\varepsilon_{D}$ is lower than that of $\varepsilon_{A}-\varepsilon_{B}$ when both strain differences are plotted against the given single axis displacement. On the other hand, a small variation of $\varepsilon_{A}-\varepsilon_{B}$ with the y-axis displacement is found in Fig. 4 (b). Given the strain gauges SG_A and SG_B are installed perfectly at the centre of the upper and lower surfaces of the shunt sheet, $\varepsilon_{A}$ and $\varepsilon_{B}$ should be zero as a result of area integration on the gauge pattern. Although the strain difference $\varepsilon_{A}-\varepsilon_{B}$ deviates from zero, the derivation can be removed by zerooffsetting during calibration. $\varepsilon_{A}-\varepsilon_{B}$ has a small sensitivity coefficient about $3 \mu \varepsilon / \mathrm{mm}$ for the y-axis displacement; the insensitivity of $\varepsilon_{A}-\varepsilon_{B}$ to the y-axis displacement leads to an accurate derivation of the $\mathrm{x}$-axis displacement.

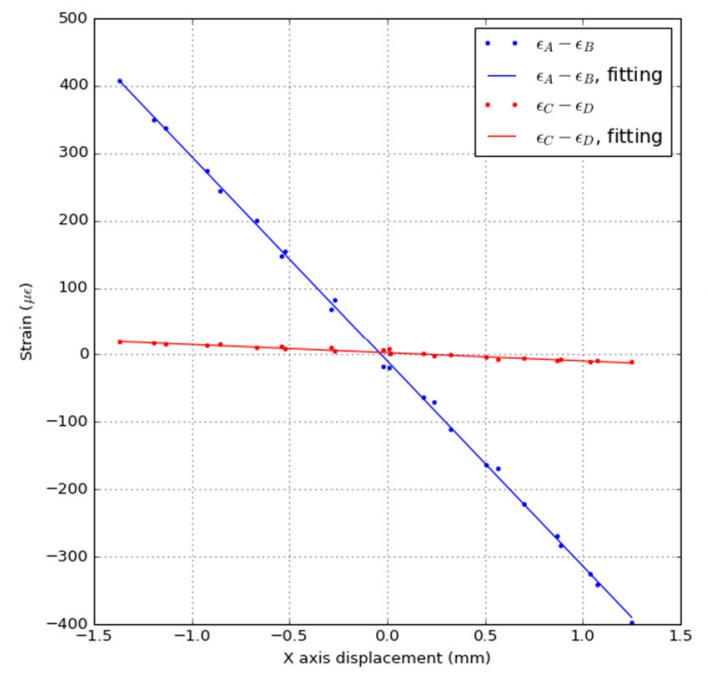

(a)

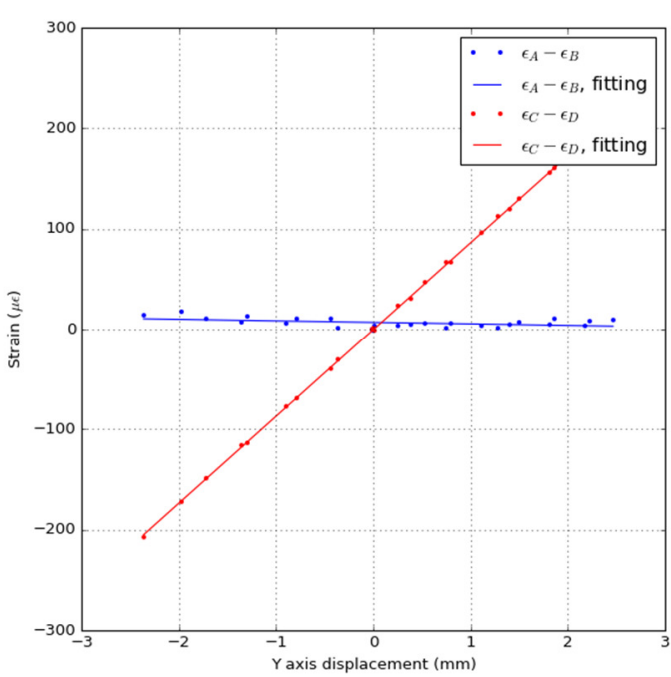

(b)

Fig. 4 Experimental measurement of strain as a function of (a) the $x$-axis displacement, and (b) the y-axis displacement. 
In addition, $\varepsilon_{A}-\varepsilon_{B}$ and $\varepsilon_{C^{-}} \varepsilon_{D}$ are designed to be insensitive to the $\mathrm{z}$-axis displacement, if all the gauges are installed in the middle of the metal sheet width. However, a weak correlation with the z-axis displacement can be observed in our experiments. The sensitivity coefficients of $\varepsilon_{A}-\varepsilon_{B}$ and $\varepsilon_{C}-\varepsilon_{D}$ to the z-axis displacement are $5 \mu \varepsilon / \mathrm{mm}$ and $6 \mu \varepsilon / \mathrm{mm}$ respectively, which are at least 10 times smaller than the sensitivity coefficient of $\varepsilon_{C^{-}} \varepsilon_{D}$ against the y-axis displacement. For the measurement of planar displacement without a significant z-axis displacement, the weak dependency of $\varepsilon_{A}-\varepsilon_{B}$ and $\varepsilon_{C^{-}} \varepsilon_{D}$ on the z-axis displacement can be ignored. Furthermore, the undesired zaxis sensitivity can be reduced by the improved gauge positioning.

The resonance frequency of the present shunt structure is obtained from the freely damping process after a manual pluck perturbation. One end of the shunt structure is fixed tightly onto a metal platform, while the other end is hanging free of constraint. After an initial y-axis force perturbation on the free end, the shunt structure is left damping in air. Strain measurement for SG_A is recorded by a wired high-speed signal conditional unit, and the sampling rate is set to 10 thousand samples per second. The first resonance frequency is obtained from the time series plot as $18.0 \mathrm{~Hz}$, as shown in Fig. 5. This experiment will be utilised to validate the modal analysis model in the finite element analysis.

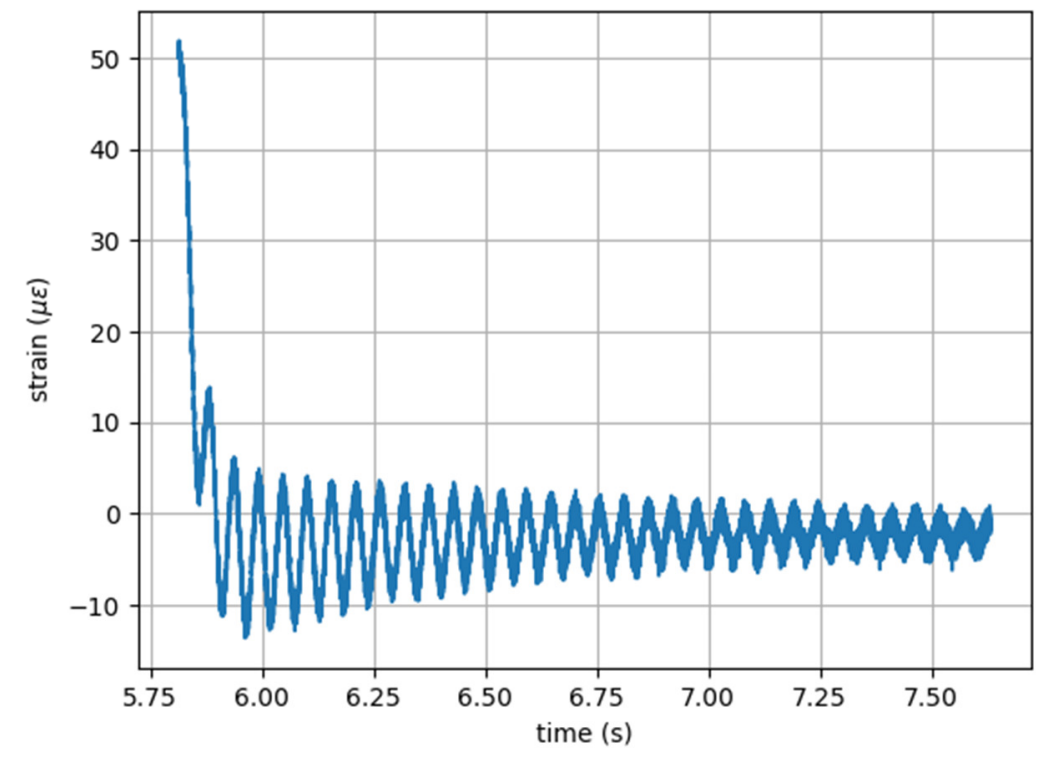

Fig. 5 Dynamic response of $\varepsilon_{\mathrm{A}}$ in a freely damping process after the manual perturbation. 


\section{Numerical analysis}

It is challenging to implement some complementary experiments, such as the study of optimal positions for strain sensor installation, dynamic response and stress concentration, and the measurement of multi-axis displacement for varying bending moments. Therefore, a complementary numerical analysis is conducted by the commercial finite element analysis software, Ansys Mechanical version 17.2. In particular, the system response to a z-axis moment is numerically investigated, and a method to distinguish strain difference $\varepsilon_{A}-\varepsilon_{B}$ induced by an X-axis displacement and a $\mathrm{z}$-axis moment is proposed.

\subsection{Validation of numerical model}

Fixed support is selected on the bottom and top surfaces of one foot to simulate the clamping fixture in our experiments, and multi-axis displacement load is applied on the bottom and top surfaces of the other end. It is required that there is a $10 \mathrm{~mm}$ distance between the clamping position and the $\cap$-shaped sections; otherwise, the clamped fillet transition will lead to different displacement sensitivities. Mesh independence test is conducted on three meshes with the element numbers of 204032 (coarse), 541885 (medium) and 1126840 (fine). It is found that the refinement from the medium to fine meshes leads to the variation of strain values less than $0.3 \%$ at the positions where the strain gauges are installed. This suggests that the medium mesh, which is shown in Fig.6, can produce mesh-independent results and thus will be used in our simulations. Furthermore, this numerical model is validated against the experimental measurement of $\varepsilon_{A}-\varepsilon_{B}$. For the x-axis displacement of $1 \mathrm{~mm}$, the simulated value of $\varepsilon_{A}-\varepsilon_{B}$ is $298 \mu \varepsilon$, while the sensitivity coefficient from the experimental fitting is $305 \mu \varepsilon / \mathrm{mm}$. The small difference between the simulated and experimental results is attributed to the deviations in gauge factor and the geometrical precision of metal sheeting process.

In addition, it is demonstrated that the one-dimensional analytical model, i.e. Eq. (14), can predict the shunt ratio. For example, the predicted shunt ratio from the analytical model is 0.000284 , which agrees with the FEA result of 0.00026 . On the other hand, a visible difference is found in warping prediction. The warping ratio predicted from the analytical model is 58.9, while the FEA gives a ratio of 65.1 . This indicates that the analytical model may under-predict the strain magnitudes on the shunt sheet by 
$10 \%$, and this small discrepancy is attributed to the negligence of fillet structure and the assumption of deflection angle in Eq. (7). However, this analytical analysis is usable to select measurement range. For the x-axis displacement of $2 \mathrm{~mm}$, the induced strains on the upper and lower centres of the shunt sheet are $303 \mu \varepsilon$ and $-293 \mu \varepsilon$ respectively, while the analytical model gives the values of $300 \mu \varepsilon$ and $-290 \mu \varepsilon$. This difference is caused by the inaccurate estimation of bending moment at the joint of the $\cap$-shaped units and the shunt sheet, see Eq. (10).

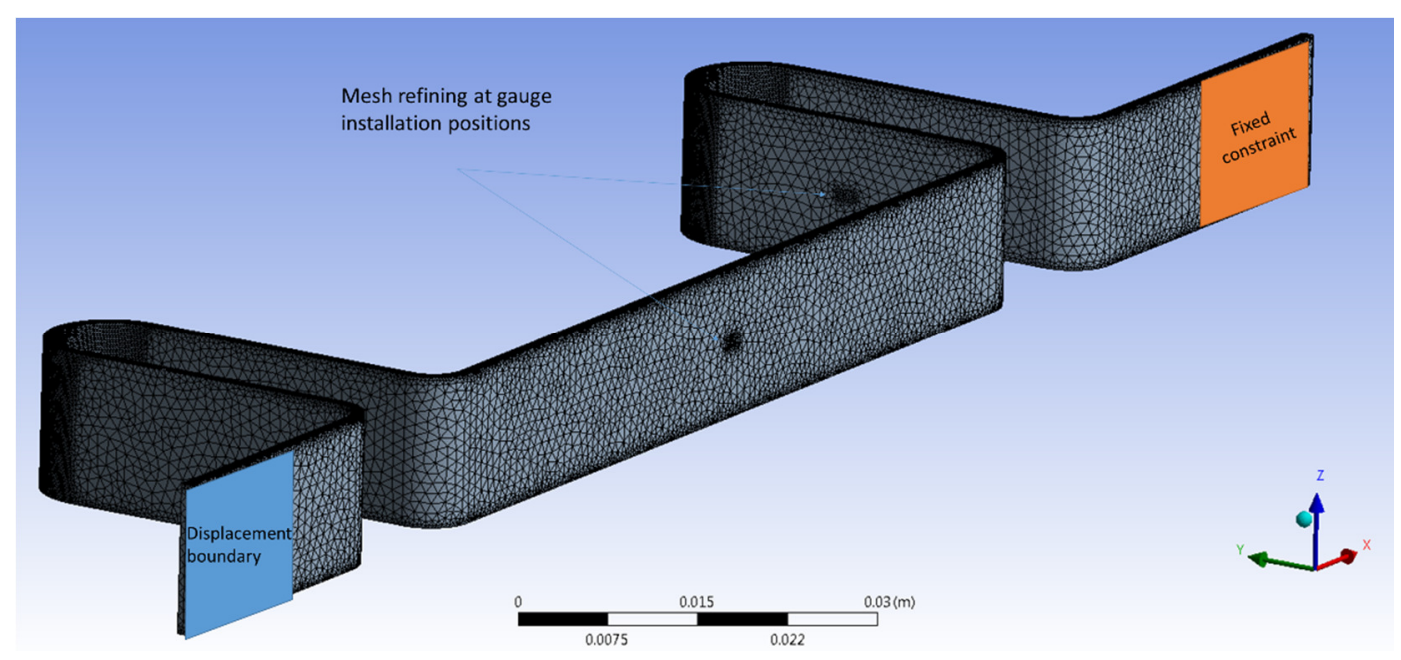

Fig. 6 The medium mesh for strain shunt design with the refinement at strain gauge installation positions

\subsection{Optimal strain gauge installation position}

The optimal strain gauge installation positions are based on the $\mathrm{x}$-axis and $\mathrm{y}$-axis strain distributions on the shunt structure surfaces with the displacement or moment applied in distinct axes (see Figs. 7 and 8). It is required that the strain gradient is low enough to minimise the impact of gauge positional errors, and at the same time the strain magnitude is high for a given axis of displacement but low in the other axes.

The centres of its upper and lower surfaces of the shunt sheet are the optimal positions for SG_A and SG_B, according to this numerical study. It can be observed from Fig. 7 (a) and (c) that the $\mathrm{x}$-axis strain gradient is small on the shunt sheet for the applied $\mathrm{x}$ axis displacement and the applied z-axis moment. The opposite sign for the $\mathrm{x}$-axis strains on the upper and lower surfaces suggests the shunt sheet is dominated by the warping deformation. A z-axis moment leads to a uniform $\mathrm{x}$-axis strain magnitude on the shunt sheet surfaces, as shown in Fig. 7 (c), and similar y-axis strain magnitudes 
are found at the positions where SG_C and SG_D are installed (see Fig. 8 (c)). With the $\mathrm{y}$-axis displacement, the $\mathrm{x}$-axis strain gradient is high on the shunt sheet where SG_A and SG_B are installed, but the X-axis strain value is zero at the shunt sheet centres (Fig. 7 (b)), indicating that $\varepsilon_{A}$ and $\varepsilon_{B}$ are both insensitive to the y-axis displacement. Therefore, $\varepsilon_{A}-\varepsilon_{B}$, as the half-bridge output for SG_A and SG_B, will be insensitive to the $\mathrm{y}$-axis displacement, but have a linear response to $\mathrm{x}$-axis displacement or the z-axis moment.

A pure y-axis displacement can be deduced from $\varepsilon_{C}-\varepsilon_{D}$ measured by a half-bridge configuration of SG_C and SG_D installed on the $\cap$-shaped units. In the current experimental setup, SG_C and SG_D are installed on the surface centres of the left and right $\cap$-shaped units (see Fig. 3 (b)). Identical strain magnitudes for SG_C and SG_D are found for the applied $\mathrm{x}$-axis displacement or $\mathrm{z}$-axis moment, as shown in Fig. 8 (a) and (c). Meanwhile, the y-axis strain induced by a pure y-axis displacement has a similar magnitude but opposite sign for SG_C and SG_D. The strain gradient is low on the flat surface of the $\cap$-shaped units for the applied y-axis displacement or $\mathrm{z}$ axis moment, giving tolerance for gauge positioning error. Therefore, a half-bridge configuration of SG_C and SG_D is ideal to sense the $y$-axis displacement, in consideration that $\varepsilon_{C}-\varepsilon_{D}$ is insensitive to $\mathrm{z}$-axis moment and $\mathrm{x}$-axis displacement. On the other hand, there exist $y$-axis strain gradients on the vertical surfaces of $\cap$-shaped units for the applied $\mathrm{x}$-axis displacement. Although a half-bridge configuration of SG_C and SG_D has the potential to cancel the $y$-axis strain induced by the $\mathrm{x}$-axis displacement, a positional error of gauge installation can lead to different strain magnitudes for SG_C and SG_D. The experimental results in Fig. 4 (a) exhibit an undesired sensitivity of $\varepsilon_{C}-\varepsilon_{D}$ to the $\mathrm{x}$-axis displacement. Therefore, it is essential to install SG_C and SG_D at the same height to minimise the sensitivity of $\varepsilon_{C}-\varepsilon_{D}$ to the $\mathrm{x}$-axis displacement. The region where the $\mathrm{y}$-axis strain is zero for varying $\mathrm{x}$-axis displacements would be the ideal position for installing SG_C and SG_D. Notably, the zero y-axis strain is not positioned at the vertical surface centre of the $\cap$-shaped units, because the fillet radius at the top is larger than that at the bottom for the current experimental setup. 


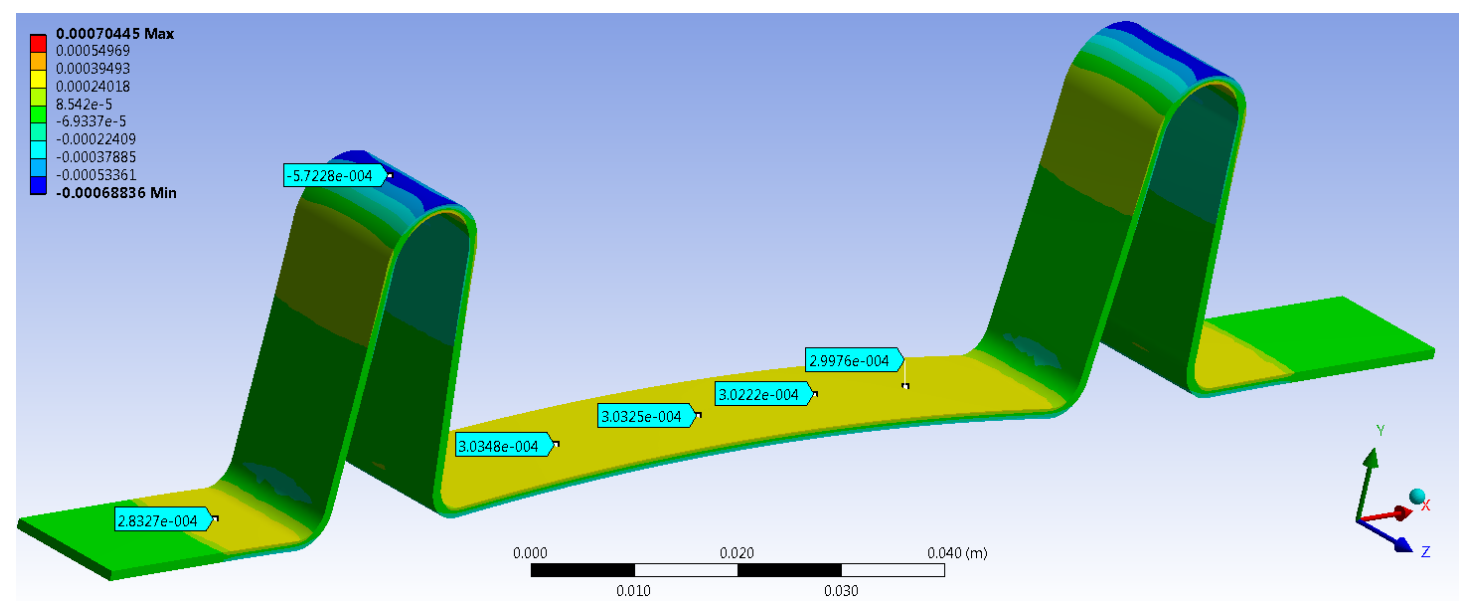

(a)

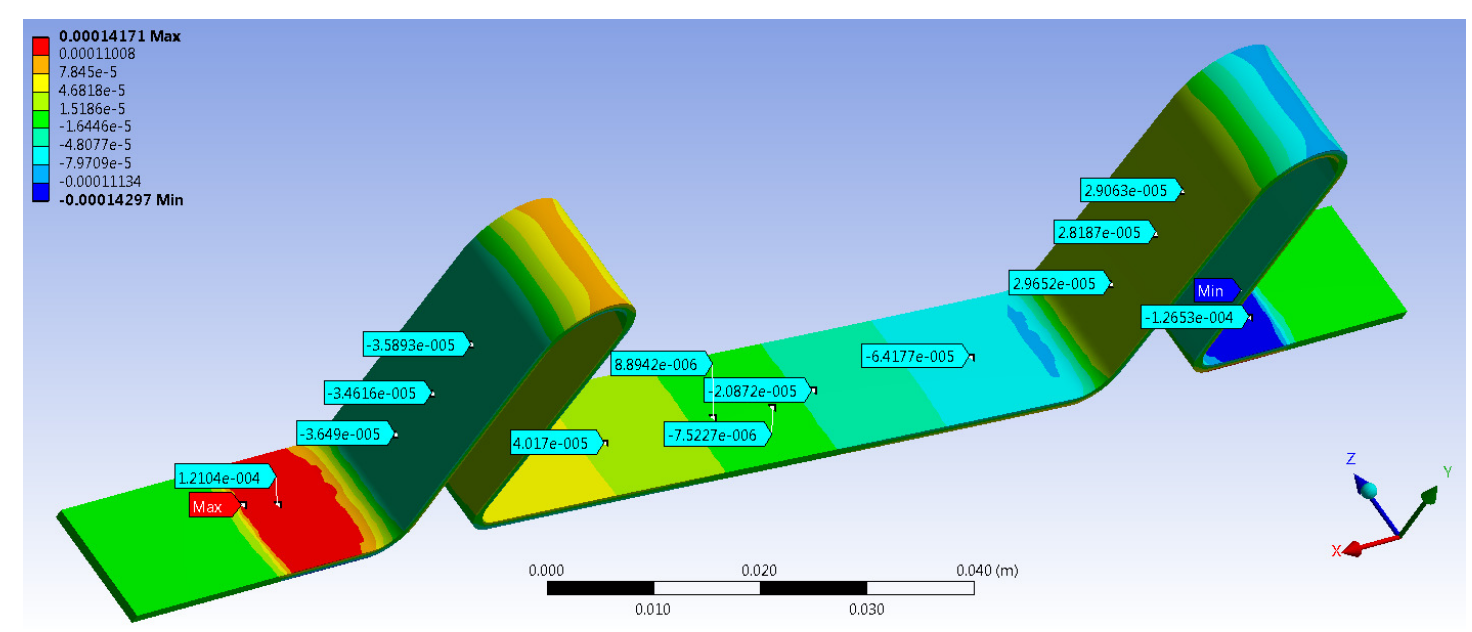

(b)

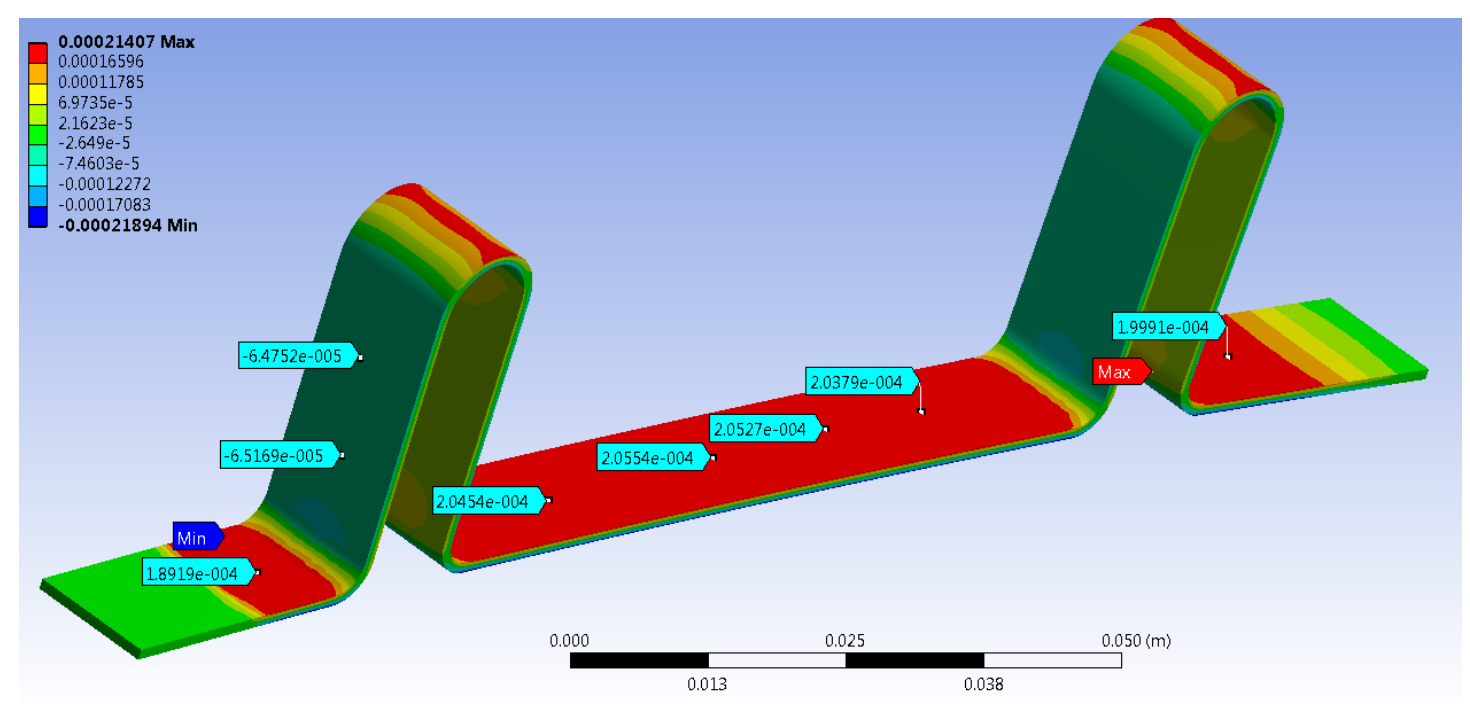

(c)

Fig. 7 x-axis normal strain contours for the structure with (a) an $x$-axis displacement $\delta_{x}$ of 2 $\mathrm{mm}$; (b) a y-axis displacement $\delta_{y}$ of $2 \mathrm{~mm}$; (c) a z-axis moment $M_{z}$ of $0.1 \mathrm{Nm}$.

Particularly, it is observed from Fig. 7 that both an $\mathrm{x}$-axis displacement and a $\mathrm{z}$-axis moment lead to a significant output of the half-bridge for $S G \_A$ and $S G \_B$, i.e. $\varepsilon_{A}-\varepsilon_{B}$. 
Judging from this half-bridge output, it is unclear whether $\varepsilon_{A}-\varepsilon_{B}$ is caused by the Xaxis displacement or by the z-axis moment acting on the sensor structure. However, it is found that a pure z-axis moment results in a magnitude of the $\mathrm{x}$-axis strain on the shunt sheet similar to that of the $y$-axis strain on the vertical surfaces of the $\cap$-shaped units, see Fig. 7(c) and Fig. 8(c). If the strain gauges SG_C and SG_D are installed on the vertical surfaces of the $\cap$-shaped units, where an $\mathrm{x}$-axis displacement leads to zero $y$-axis strain, quarterly bridge measurement of SG_C and SG_D can distinguish the $\mathrm{z}$-axis moment from the $\mathrm{x}$-axis displacement. The value of $\varepsilon_{C}+\varepsilon_{D}$ should be proportional to the $\mathrm{z}$-axis moment applied, but insensitive to the $\mathrm{x}$-axis displacement. From the $y$-axis strain magnitudes of SG_C and SG_D, the applied z-axis moment can be derived without confusion. In practice, 2 extra strain gauges that form a half-bridge are preferred for the z-axis moment measurement, since SG_C and SG_D are reserved for the $y$-axis displacement measurement through a half-bridge configuration.

These extra strain gauges, denoted as SG_E and SG_F in Fig. 2 (b), can distinguish $\varepsilon_{A}-\varepsilon_{B}$ induced by a z-axis moment from that incurred by an $\mathrm{x}$-axis displacement. The strain gauges at the optimal positions on the $\cap$-shaped units will have a zero $y$-axis strain magnitude for a pure $\mathrm{x}$-axis displacement and a similar magnitude for a pure $\mathrm{y}$ axis displacement. A half-bridge consisting of SG_E and SG_F is advantageous over the quarterly bridge for thermal strain cancelation, and its output is only sensitive to a z-axis moment. Thereby, a high half-bridge output $\varepsilon_{E^{-}} \varepsilon_{F}$ occurs when a z-axis moment is applied on the shunt structure, while near zero output is expected for a pure y-axis displacement or an $\mathrm{x}$-axis displacement. Once the z-axis moment is deduced from $\varepsilon_{E^{-}}$ $\varepsilon_{F}$ measurement and its sensitivity coefficient calibrated against varying $\mathrm{z}$-axis moments, the portion of $\varepsilon_{A}-\varepsilon_{B}$ induced by the z-axis moment can be subtracted from total $\varepsilon_{A}-\varepsilon_{B}$ variation. Eventually, the applied $\mathrm{x}$-axis displacement can be determined from the resultant portion of $\varepsilon_{A}-\varepsilon_{B}$ induced by the pure $\mathrm{x}$-axis displacement. The derivation of multi-axis displacement will be further demonstrated in Section 4.3. 


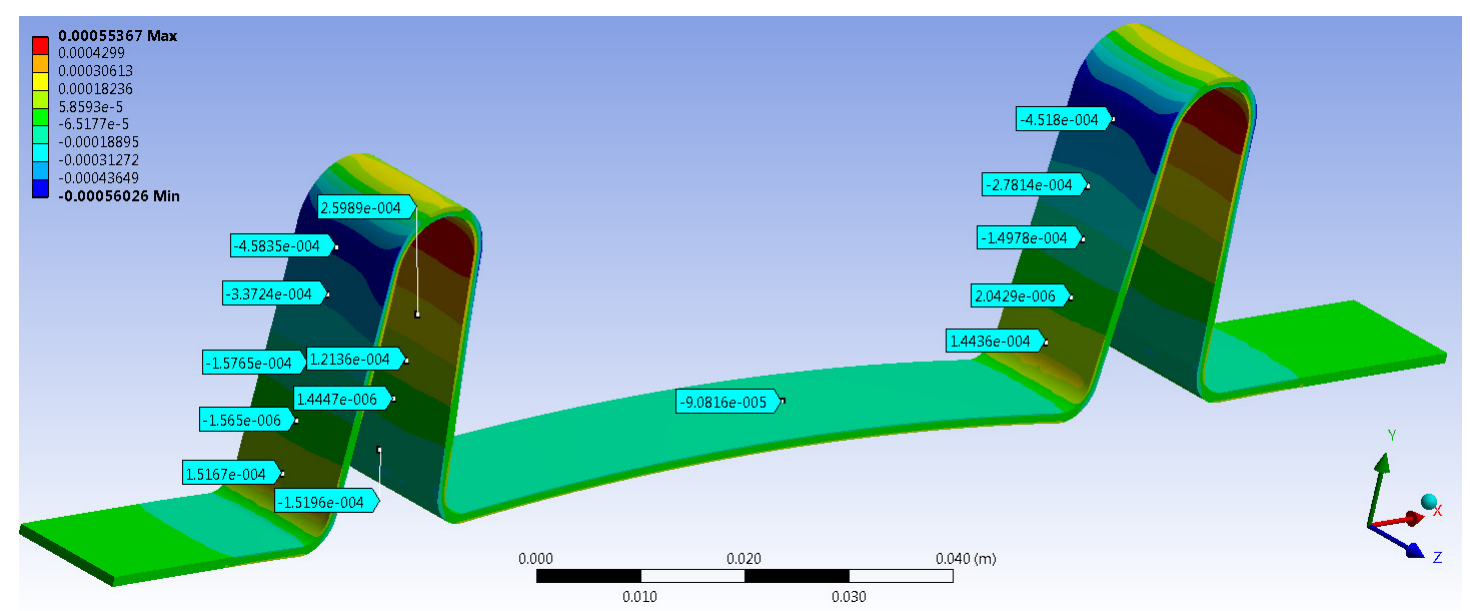

(a)

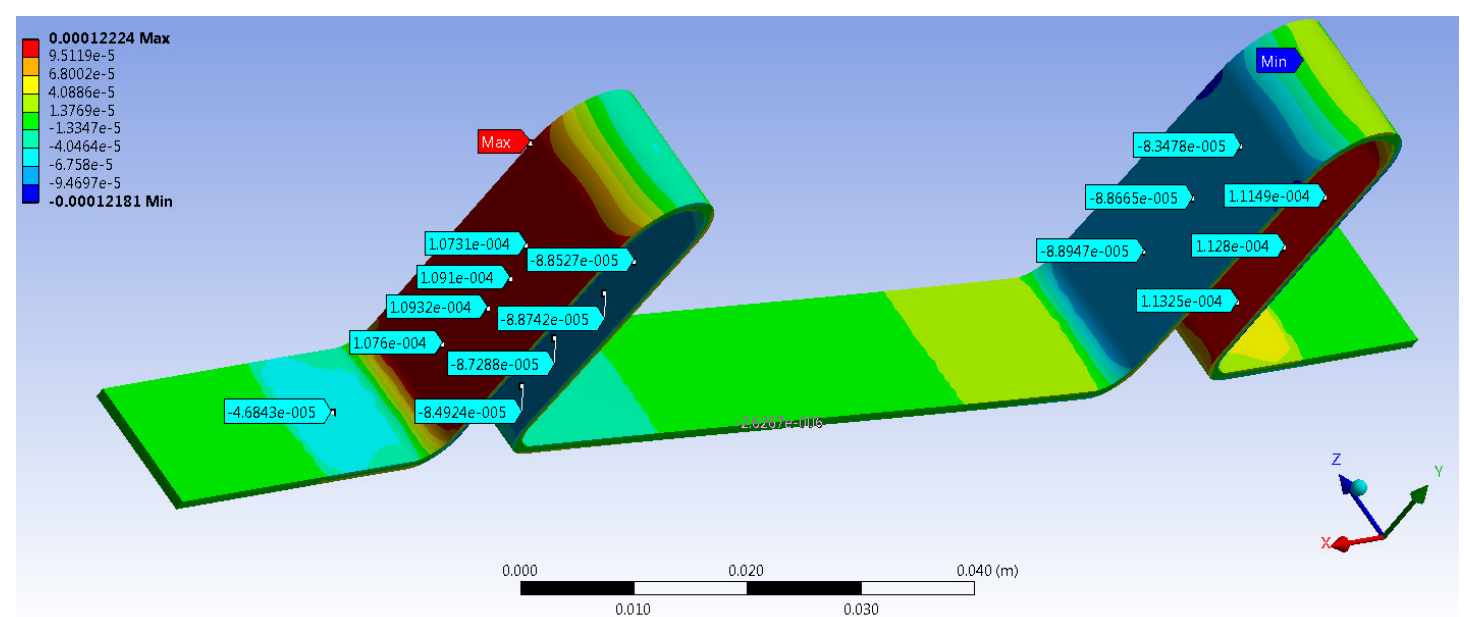

(b)

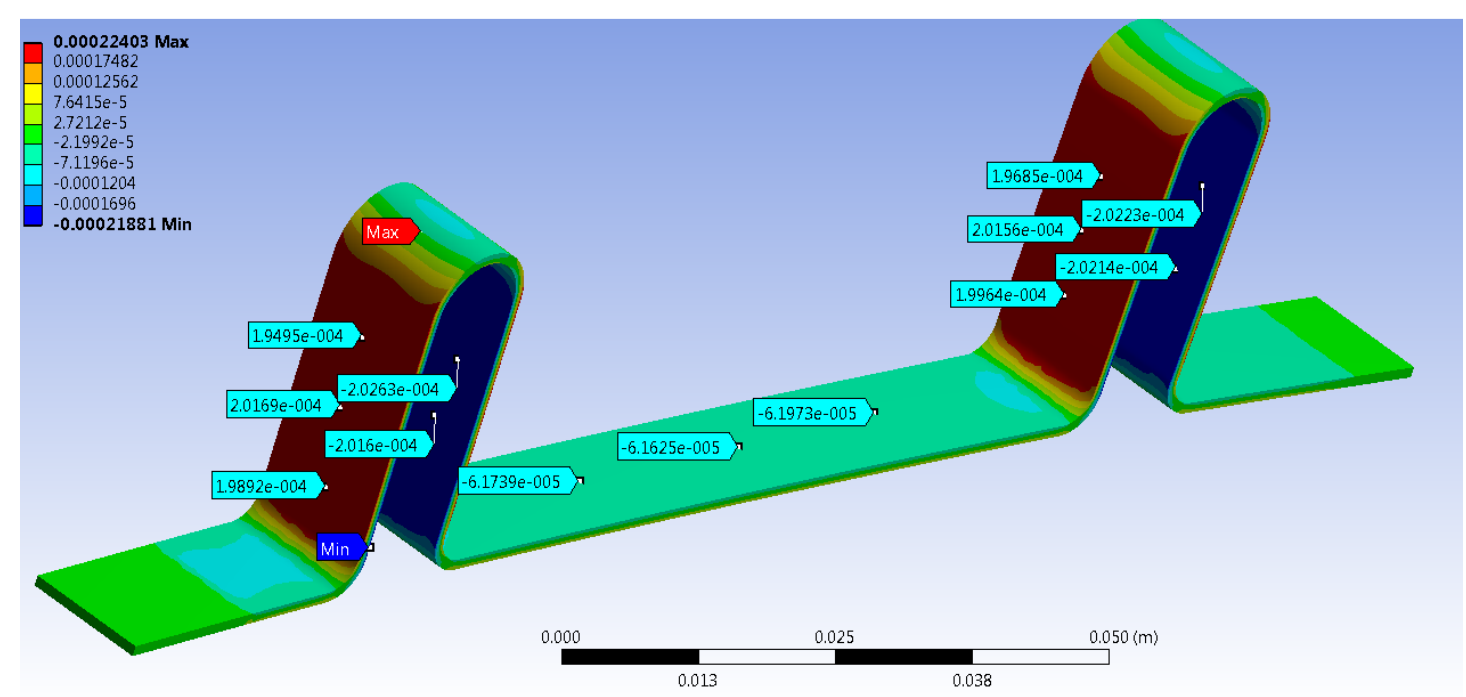

(c)

Fig. 8 y-axis normal strain contours for the structure with (a) an $x$-axis displacement $\delta_{x}$ of 2 $\mathrm{mm}$; (b) a y-axis displacement $\delta_{y}$ of $2 \mathrm{~mm}$; (c) a z-axis moment $M_{z}$ of $0.1 \mathrm{Nm}$. 
In summary, the general guidelines for the optimal gauge installation position are given as follows. First of all, gauges must be installed in the middle of the sheet width, to avoid the sensitivity to the z-axis displacement or $\mathrm{x}$-axis/y-axis moment. Secondly, gauges should be installed on the flat surface where the strain gradient is low for the given single axis displacement. Usually, it is near or in the middle of the flat surfaces. Extra strain gauges should be installed on other flat surfaces of the shunt structure, to avoid gauge overlapping. Finally, the gauge measurement should give a large strain magnitude for the displacement in a specific axis but nearly zero in the other axes. For example, the half-bridge output of SG_A and SG_B gives the maximum output for Xaxis displacement, but zero for the $y$-axis displacement. This configuration can increase the signal-to-noise ratio, in which the noise can be incurred by the positional errors of gauge or geometrical errors of shunt structure.

\subsection{Response to multi-axis displacement}

Given multiple strain gauges at the optimal positions, the combination of $\mathrm{x}$-axis and $y$-axis displacements with z-axis moment applied at one end of the shunt structure can be measured simultaneously. Table 1 demonstrates the response of each gauge to the combination of displacement and moment. Stain gauges from SG_A to SG_D are located in the flat surface centres for the current experiment setup, while the proposed extra measurement positions by SG_E and SG_F in our numerical study have no corresponding gauges in the experiment. When the gauge positioning error is absent in the numerical simulation, an ideal linear response is exhibited for $\varepsilon_{A}-\varepsilon_{B}$ against the applied pure $\mathrm{x}$-axis displacements, while $\varepsilon_{A}$ and $\varepsilon_{B}$ are not sensitive to pure y-axis displacements, see case \#1 to \#5 in Table 1. In addition, both $\mathrm{x}$-axis and $\mathrm{y}$-axis displacements are found to affect $\varepsilon_{C}$ and $\varepsilon_{D}$, but the half-bridge output of SG_C and SG_D, i.e. $\varepsilon_{C^{-}} \varepsilon_{D}$, only depends on the y-axis displacement. Finally, in the absence of $\mathrm{z}$-axis moment, the combination of $\mathrm{x}$-axis and $\mathrm{y}$-axis components can be measured by $\varepsilon_{A}-\varepsilon_{B}$ and $\varepsilon_{C}-\varepsilon_{D}$ independently, which can be seen from the case \#6 in Table 1. Note that all the mentioned gauge outputs should be insensitive to the z-axis displacement, as well as the $\mathrm{x}$-axis and $\mathrm{y}$-axis moments, if all gauges are perfectly installed at the middle of sheet width.

According to Table 1, $\varepsilon_{\mathrm{A}}$ and $\varepsilon_{\mathrm{B}}$ are sensitive to both the $\mathrm{x}$-axis displacement and the $\mathrm{Z}$-axis moment, and thus the contribution from both actuations must be distinguished. 
As discussed in the previous section, an extra half-bridge consisting of SG_E and SG_F should be introduced to measure the applied z-axis moment. SG_E and SG_F should be installed on the $\cap$-shaped stress relieving units where a pure $\mathrm{x}$-axis displacement results in zero strain for both $\varepsilon_{\mathrm{E}}$ and $\varepsilon_{\mathrm{F}}$. For the case \#8 in Table 1, a pure z-axis moment leads to similar strain magnitudes for $\varepsilon_{\mathrm{A}}$ and $\varepsilon_{\mathrm{B}}$ on the shunt sheet and $\varepsilon_{\mathrm{E}}$ and $\varepsilon_{\mathrm{F}}$ on the stress relieving units; an approximation of the pure z-axis moment induced strain magnitude on SG_A and SG_B by that on SG_E and SG_F is acceptable in consideration of their discrepancy as low as $2 \%$. Therefore, a full bridge configuration, where SG_A and SG_E act as the first half-bridge and SG_B and SG_F as the second, can measure the $\mathrm{x}$-axis displacement. The output of the full bridge, $\left(\varepsilon_{\mathrm{A}^{-}}\right.$ $\left.\varepsilon_{\mathrm{E}}\right)-\left(\varepsilon_{\mathrm{B}}-\varepsilon_{\mathrm{F}}\right)$, which is not sensitive to the $\mathrm{z}$-axis moment, is proportional to the pure $\mathrm{x}-$ axis displacement. Alternatively, the $\mathrm{x}$-axis displacement can be computed by a signal processing unit, once the z-axis moment applied on the sensor can be derived from $\varepsilon_{\mathrm{E}}-$ $\varepsilon_{F}$ measurement and its pre-calibrated sensitivity coefficient. Given the sensitivity coefficients with respect to pure $\mathrm{x}$-axis displacements and pure $\mathrm{z}$-axis moments, $\varepsilon_{\mathrm{A}}-\varepsilon_{\mathrm{B}}$ subtracting the portion induced by the $\mathrm{z}$-axis moment can be used to deduce the $\mathrm{x}$-axis displacement, see case \#9 in Table 1.

Table 1 Response of the shunt structure to compound $x$-axis and $y$-axis displacements

\begin{tabular}{|l|l|l|l|l|l|l|l|l|l|l|}
\hline $\begin{array}{l}\text { Case } \\
\text { ID }\end{array}$ & $\begin{array}{l}\delta_{\mathrm{x}} \\
(\mathrm{mm})\end{array}$ & $\begin{array}{l}\delta_{\mathrm{y}} \\
(\mathrm{mm})\end{array}$ & $\begin{array}{l}\delta_{\mathrm{z}} \\
(\mathrm{mm})\end{array}$ & $\begin{array}{l}\mathrm{M}_{\mathrm{z}} \\
(\mathrm{Nm})\end{array}$ & $\begin{array}{l}\varepsilon_{\mathrm{A}} \\
(\mu \varepsilon)\end{array}$ & $\begin{array}{l}\varepsilon_{\mathrm{B}} \\
(\mu \varepsilon)\end{array}$ & $\begin{array}{l}\varepsilon_{\mathrm{C}} \\
(\mu \varepsilon)\end{array}$ & $\begin{array}{l}\varepsilon_{\mathrm{D}} \\
(\mu \varepsilon)\end{array}$ & $\begin{array}{l}\varepsilon_{\mathrm{E}} \\
(\mu \varepsilon)\end{array}$ & $\begin{array}{l}\varepsilon_{\mathrm{F}} \\
(\mu \varepsilon)\end{array}$ \\
\hline$\# 1$ & 1 & 0 & 0 & 0 & 152 & -146 & -78.0 & -78.0 & 0 & 0 \\
\hline$\# 2$ & 2 & 0 & 0 & 0 & 303 & -293 & -156 & -156 & 0 & 0 \\
\hline$\# 3$ & -2 & 0 & 0 & 0 & -303 & 293 & 156 & 156 & 0 & 0 \\
\hline$\# 4$ & 0 & 2 & 0 & 0 & 0 & 0 & -88.3 & 88.3 & 109 & 109 \\
\hline$\# 5$ & 0 & -2 & 0 & 0 & 0 & 0 & 88.3 & -88.3 & -109 & -109 \\
\hline$\# 6$ & 2 & 2 & 0 & 0 & 303 & -293 & -244 & 67.3 & 109 & 109 \\
\hline$\# 7$ & 0 & 0 & 1 & 0 & 0 & 0 & 0 & 0 & 0 & 0 \\
\hline$\# 8$ & 0 & 0 & 0 & 0.1 & 205 & -205 & -201 & -201 & 201 & -201 \\
\hline$\# 9$ & 1 & 0 & 0 & 0.1 & 357 & -351 & -279 & -279 & 201 & -201 \\
\hline
\end{tabular}

\subsection{Evaluation of stress concentration}

The maximum displacement for a given axis that a shunt structure can measure is limited by the elastic limit (i.e. yielding strength) of the sheet material and the ratio of the maximum strain on the structure to that measured by gauges. In order to guarantee 
a linear output, the proportionality limit of the shunt material should not be exceeded. Furthermore, the maximum stress on the loaded structure should be less than the yielding strength to avoid plastic deformation, ideally below the fatigue limit of the sheet material if a highly dynamic displacement is to be measured. Stress concentration, which limits the displacement measurement range, should be reduced. Fillet transition has been confirmed to effectively reduce the stress concentration. Fig. 9 illustrates the von-Mises stress contours on the surface of the shunt structure with an $\mathrm{x}$-axis displacement of $0.002 \mathrm{~m}$. The von-Mises equivalent stress is as low as $85 \mathrm{MPa}$ at the centre of shunt sheet bottom surface, where SG_B is installed. The maximum stress of $137 \mathrm{MPa}$, observed at the top of the $\cap$-shape turns, is lower than the typical yielding strength of medium carbon steel of $350 \mathrm{MPa}$ and also within the fatigue limit of the carbon steel material (40\% of the yielding strength) [17]. Like other sensors based on the strain measurement, the input overload could cause plastic deformation of the strain relieving units and thus a shift of zero offset. For a long-term displacement measurement, zero-offsetting should be done periodically against baseline sensors, similar to other strain gauge based sensors. Notably, the amplitude of dynamic displacement can be measured precisely if the yielding stress limit is not surpassed, since the sensitivity of the present displacement sensor relies only on the geometrical parameters of shunt structure and is less affected by the shifted zero offset.

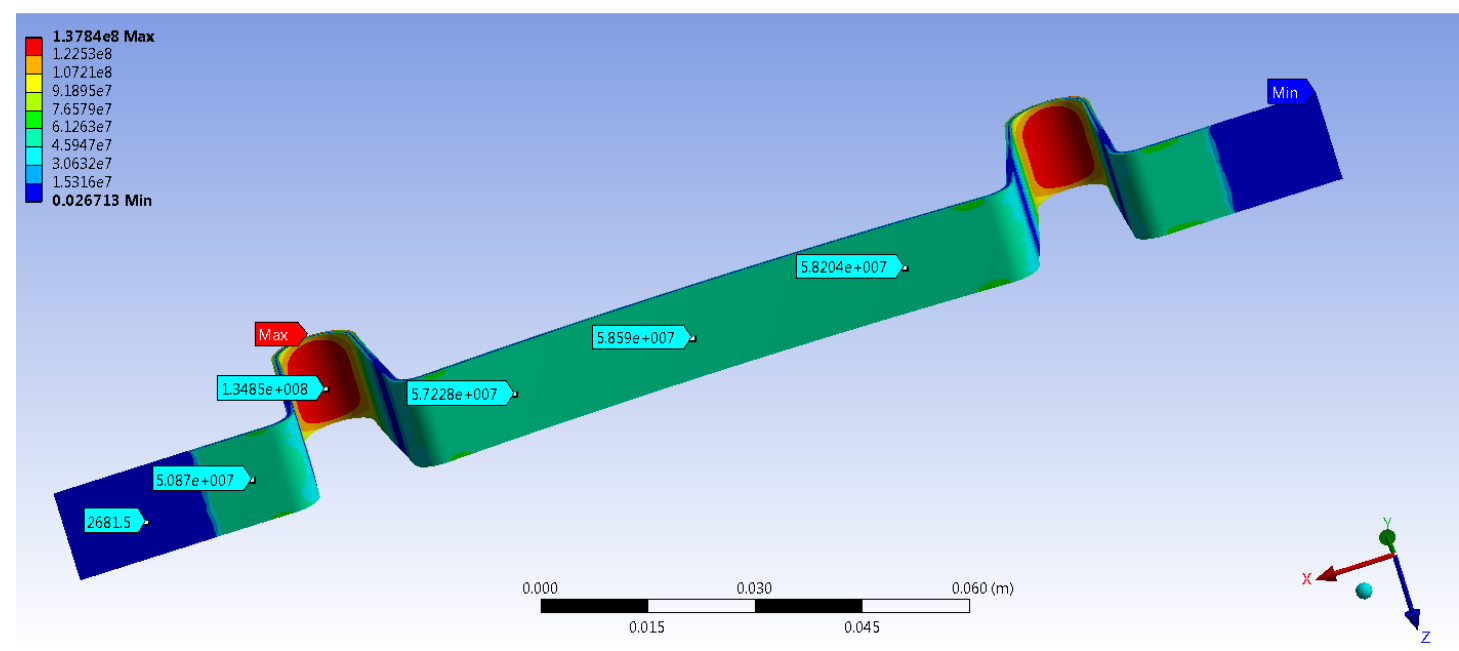

Fig. 9 Equivalent (von-Mises) stress distribution on the surface of strain shunt for an x-axis displacement of $0.002 \mathrm{~m}$ 
For the dynamic displacement sensor based on strain shunt structure, the fatigue failure of fixture/fastening is another major concern for long-term deployment. In the current experimental setup, the shunt structure is fastened to the target surface by screws. Welding can be employed to bond this displacement sensor onto metallic surfaces, while adhesive is a choice for non-metallic targeted surfaces. The failure of sensor fixture caused by weathering or corrosion is worth of further investigation.

\subsection{Modal analysis of dynamic response}

The dynamic response of the displacement sensor based on the strain shunt is investigated numerically. In the experimental evaluation, a characteristic frequency of $18.0 \mathrm{~Hz}$ is found in freely damping with a y-axis perturbation force. Meanwhile, the numerical study gives a similar value of $18.5 \mathrm{~Hz}$ for the experiment-matched boundary conditions: both $\mathrm{x}$-axis and $\mathrm{y}$-axis free of constraint at one end while the other end fixed. Modal analysis indicates that the values of the first natural frequency are respectively $83.9 \mathrm{~Hz}, 28.8 \mathrm{~Hz}$ and $45.8 \mathrm{~Hz}$ for the $\mathrm{x}$-axis, $\mathrm{y}$-axis, and z-axis free of constraint, if the other degrees of freedom are fixed. These natural frequencies are much higher than the first harmonic frequency of freely damping process. The dynamic displacement, typically found in the crack width variation in structural health monitoring, can be captured by the present innovative displacement sensor. In addition, decreasing the shunt sheet length can significantly increase the first resonance frequency at the cost of the reduced displacement measurement range.

\section{Conclusion}

In this paper, a displacement sensor based on the strain shunt structure is proposed and validated experimentally. The working principle of the displacement measurement using strain gauges on a strain shunt structure is illustrated, and the sensitivity of displacement is derived analytically from a simplified one-dimensional model.

The prototype sensor has been experimentally tested for static displacement in multiple axes, and the analytical prediction shows an excellent agreement with the experimental result. A constant sensitivity, i.e. the linear relationship between the strain gauge half-bridge output and the $\mathrm{x}$-axis or $\mathrm{y}$-axis displacement between two fixing points, is confirmed by the experiments. Although the sensitivity coefficients 
are axis-dependent, the measurement of multi-axis displacement is feasible for the independent half-bridges output of strain gauges at the optimal installation positions. Utilising multiple strain gauges, the displacements in $\mathrm{x}$-axis and $\mathrm{y}$-axis can be measured simultaneously.

The optimal gauge positioning is investigated by finite element analysis, and it is found that the strain gauges should be installed in the middle of the metal sheet width, on the flat surface where strain gradient is low, and in the place where the half-bridge gauge output is high for the displacement in one axis but low or zero for the others. Furthermore, the numerical study has suggested that an extra strain gauge pair can distinguish the shunt sheet deformations induced by a $\mathrm{z}$-axis moment and a $\mathrm{x}$-axis displacement.

\section{Conflict of Interest}

An industrial patent, METHODS AND APPARATUS FOR MEASURING

DEFORMATOIN, GB/1714558.2, was filed by the first author, Qingfeng Xia, on Sep. $11,2017$.

\section{Acknowledgement}

This work is supported by the National Natural Science Foundation of China (Nos. 51506168 and 51711530130), the National Key Research and Development Project of China (No. 2016YFB0200902), and the China Postdoctoral Science Foundation (No. 2016M590943). H. Liu gratefully acknowledges the financial supports from Thousand Youth Talents Program for Distinguished Young Scholars, and the Young Talent Support Plan of Xi'an Jiaotong University.

\section{Nomenclature}

\begin{tabular}{|l|l|}
\hline Symbol & \\
\hline A & Cross section area $\left(\mathrm{m}^{2}\right)$ \\
\hline b & Thickness of the shunt sheet $(\mathrm{m})$ \\
\hline E & Elastic modulus $(\mathrm{Pa})$ \\
\hline F & Force $(\mathrm{N})$ \\
\hline f & Frequency $(\mathrm{Hz})$ \\
\hline H & Height $(\mathrm{m})$ \\
\hline I & Second moment of inertia for cross-section \\
\hline
\end{tabular}




\begin{tabular}{|l|l|}
\hline $\mathrm{k}$ & Stiffness of spring $\left(\mathrm{kg} / \mathrm{s}^{2}\right)$ \\
\hline $\mathrm{L}$ & Length $(\mathrm{m})$ \\
\hline $\mathrm{M}$ & Moment $(\mathrm{Nm})$ \\
\hline $\mathrm{m}$ & Mass $(\mathrm{kg})$ \\
\hline $\mathrm{r}$ & ratio \\
\hline $\mathrm{W}$ & Width of $\cap$-shaped cantilever $(\mathrm{m})$ \\
\hline $\mathrm{w}$ & Width of the shunt sheet $(\mathrm{m})$ \\
\hline $\mathrm{X}, \mathrm{y}, \mathrm{Z}$ & Coordinate $(\mathrm{m})$ \\
\hline$\delta$ & Displacement $(\mathrm{m})$ \\
\hline$\theta$ & Angle $(\mathrm{rad})$ \\
\hline$\varepsilon$ & Strain \\
\hline Subscript & \\
\hline $\mathrm{c}$ & Cantilever of the strain shunt \\
\hline $\mathrm{s}$ & Shunt sheet of the strain shunt \\
\hline X,y,Z & Axis of Cartesian coordinate \\
\hline $\begin{array}{l}\text { A,B,C, } \\
\text { D,E,F }\end{array}$ & Name of strain gauge \\
\hline
\end{tabular}

\section{Reference}

1. Segovia Garcia, M.D.C., M.J.T. Revie, and F. Quail. Condition monitoring data in the study of offshore wind turbines' risk of failure. 2013. ASME.

2. Ciang, C.C., J.-R. Lee, and H.-J. Bang, Structural health monitoring for a wind turbine system: a review of damage detection methods. Measurement Science and Technology, 2008. 19(12): p. 122001.

3. Hamilton, A. and F.J. Quail, Detailed state of the art review for the different on-line/in-line oil analysis techniques in context of wind turbine gearboxes. Journal of Tribology, 2011. 133(4).

4. Fukuda, Y., et al., Vision-based displacement sensor for monitoring dynamic response using robust object search algorithm. Sensors Journal, IEEE, 2013. 13(12): p. 4725-4732.

5. $\quad \mathrm{Ng}, \mathrm{T}$., The optical mouse as a two-dimensional displacement sensor. Sensors and Actuators A: Physical, 2003. 107(1): p. 21-25.

6. Xia, Q. and L. Yan, Application of wireless power transfer technologies and intermittent energy harvesting for wireless sensors in rotating machines.

Wireless Power Transfer, 2016.

7. $\quad$ Valdevit, A., et al., Displacement transducer. 2004, Google Patents.

8. Micromeasurement, V., Linear Displacement Sensors, V. Micromeasurement, Editor. 2016, Vishay Micromeasurement. p. 1-3.

9. Mohammad, I. and H. Huang, Monitoring fatigue crack growth and opening using antenna sensors. Smart Materials and Structures, 2010. 19(5): p. 055023.

10. Pandey, N.K. and B.C. Yadav, Embedded fibre optic microbend sensor for measurement of high pressure and crack detection. Sensors and Actuators A: Physical, 2006. 128(1): p. 33-36.

11. Valença, J., et al., Automatic crack monitoring using photogrammetry and image processing. Measurement, 2013. 46(1): p. 433-441. 
12. Gao, T. and Q. Xia, Experimental and numerical study of a shunt structure for large dynamic strain measurement. IEEE Sensors Journal, 2016. 16(23): p. 8403-8411.

13. Qingfeng Xia, F.Q., Principles and validation of strain gauge shunt design for large dynamic strain measurement. Sensors and Actuators A Physical, 2016. 241.

14. Yaralioglu, G., et al., Analysis and design of an interdigital cantilever as a displacement sensor. Journal of Applied Physics, 1998. 83(12): p. 7405-7415.

15. Bauchau, O. and J. Craig, Euler-Bernoulli beam theory, in Structural Analysis. 2009, Springer. p. 173-221.

16. Juvinall, R.C. and K.M. Marshek, Fundamentals of machine component design. 4th ed. 2006: J. Wiley (New York).

17. Soboyejo, W., Mechanical properties of engineered materials. Vol. 152. 2002: CRC press. 